\title{
Development of powerful Ka-band FEM-amplifiers with broad frequency tuning
}

\author{
A.K. Kaminsky $^{1}$, I.V. Bandurkin ${ }^{2}$, D.E. Donets ${ }^{1}$, S.V. Kuzikov ${ }^{2}$, \\ N.Yu. Peskov², A.V. Savilov², S.N. Sedykh ${ }^{1}$, A.A. Vikharev ${ }^{2}$ \\ ${ }^{1}$ Joint Institute for Nuclear Research, Dubna, Russia, alim_kaminsky@mail.ru \\ ${ }^{2}$ Institute of Applied Physics RAS, Nizhny Novgorod, Russia
}

\section{Introduction}

During the several preceding years, an oscillator scheme of a free electron maser (FEM) has been realized in the joint experiments between JINR (Dubna) and IAP RAS (Nizhny Novgorod) [1]. The project was aimed at accelerating applications, and the FEM operates in a frequency range of $30 \mathrm{GHz}$ and generates $20 \mathrm{MW} / 200 \mathrm{~ns}$ pulses with a spectrum width of 6-7 MHz, which is close to the theoretical limit. The high output power and stability of a narrow-band operation regime allowed this JINRIAP FEM to be used in a number of actual demand applications, including testing the components of highgradient accelerators of the next generation [2].

However, many potential applications of powerful microwave sources require an RF-radiation phase to be controlled. Moreover, some applications need a broad instantaneous amplification band or a fast frequency tuning. In particular, for accelerating applications, precise matching of the radiation frequency with the frequency of high-gradient structure should be provided. For this purpose, the FEM-amplifier project is developed based on LIU-3000.

\section{FEM in a grazing incident regime}

Two regimes of Ka-band wave amplification have been simulated. In the first one, the electrons move along the uniform helical wiggler with the axial velocity equal to the group velocity of the operating wave (grazing regime) (Fig. 1). Provided the FEM resonance condition $\omega=\left(2 \pi / d_{\mathrm{w}}+h\right) v_{\|}$is fulfilled, an efficient electron-wave coupling in the wide band of the operating frequencies takes place. In simulations, the possibility to provide a wide instant amplification band and, thus, fast control over the operation frequency, as well as a high efficiency and a high output power, were demonstrated. For the driving power of $4-5 \mathrm{~kW}$, the simulations predicted an amplification of up to $35 \mathrm{~dB}$ in the range of $2-32 \mathrm{GHz}$ saturating at approximately $1 \mathrm{~m}$ of the interaction space.

Experimental study of this scheme of the FEMamplifier was performed at the LIU-3000 accelerator. A magnetron tunable in the range of $29.5-30.5 \mathrm{GHz}$ was used to supply an input signal of about $4 \mathrm{~kW}$ power at the entrance of the interaction space. Circular-polarization of the driving wave-beam was provided by the section of elliptical waveguide.

In the first series of the experiments, a "regular" helical wiggler of $6-\mathrm{cm}$ period and transverse field of $0.1-$ $1.1 \mathrm{~T}$ was used to pump the operating transverse velocity into the electron beam focused by an axial magnetic field of around $0.15 \mathrm{~T}$ in a reversed guide field regime. To achieve a high efficiency of the FEM operation, an im- proved construction of pulsed helical wiggler was developed. The regular part of the wiggler represents a double bifilar spiral with winding currents of the same direction spaced apart to an angle of $60^{\circ}$ (Fig. 2). Such optimization of the winding geometry ensures a high transverse homogeneity of the field inside the wiggler compared with the single bifilar wiggler. The wiggler entrance was slowly up-tapered over the initial six periods in order to provide the excitation of operating transverse oscillations in the beam, and down-tapered over the last three periods for a better beam transportation at the wiggler output.

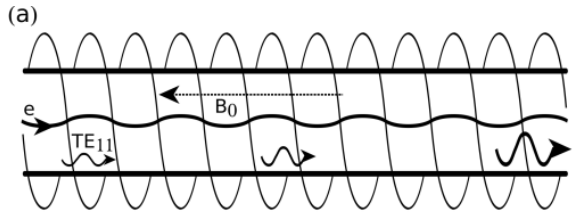

(b)

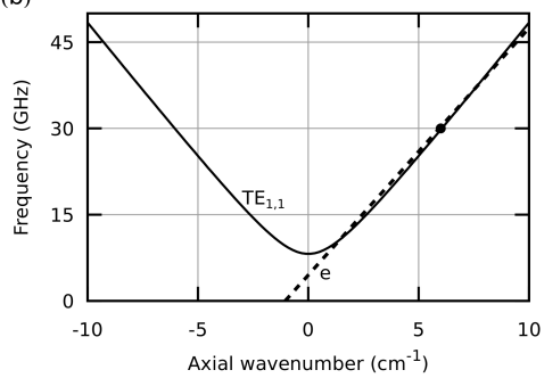

Fig. 1. (a) Schematic of the FEM amplifier with a helical wiggler and reverse guide field and (b) dispersion diagram for the 30-GHz JINR-IAP FEM-amplifier operated in the grazing regime. Solid and dashed lines show the dispersion of the $\mathrm{TE}_{1,1}$ wave and the electron beam, respectively

During the experiments, the stable regime of input signal amplification was observed in the designed range of parameters (Fig. 3). The FEM was tuned to the maximum output power by changing the wiggler and solenoid fields, as well as by small variation of the particle energy. In optimal regime $\left(B_{0} \approx-1.5 \mathrm{kG}, B_{\mathrm{w}} \approx 1-1.2 \mathrm{kG}\right)$, according to the calorimeter measurements, the radiation power achieved 17-20 MW at the operating frequency band around $30 \mathrm{GHz}$, in good agreement with the simulations. It should be noted that in the experiments, an about 10 $15 \%$ current loss was observed in the region where the electron beam formed by the accelerator was injected into the wiggler. As a result, the beam current in the interaction space was of the level taken in the simulations. The output pulse length was near $200 \mathrm{~ns}$, which is close to the total duration of the beam pulse.

The spectrum width of the amplified signal amounted to 6-7 MHz, which is close to the natural spectrum width for the observed pulse duration. For the fixed beam and 
electron-optical system parameters, this level of output power was observed within the whole band of the magnetron frequency tuning of $\pm 0.5 \mathrm{GHz}$, which models the instant amplification band [5]. At the same time, an increase in the wiggler field up to $1.3 \mathrm{kG}$ resulted in selfexcitation of the system at parasitic frequencies.
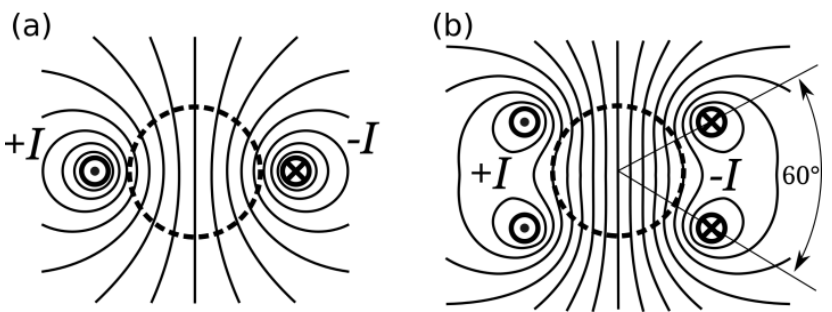

Fig. 2. Transverse magnetic field distribution inside a bifilar $(a)$ and double bifilar $(b)$ undulators
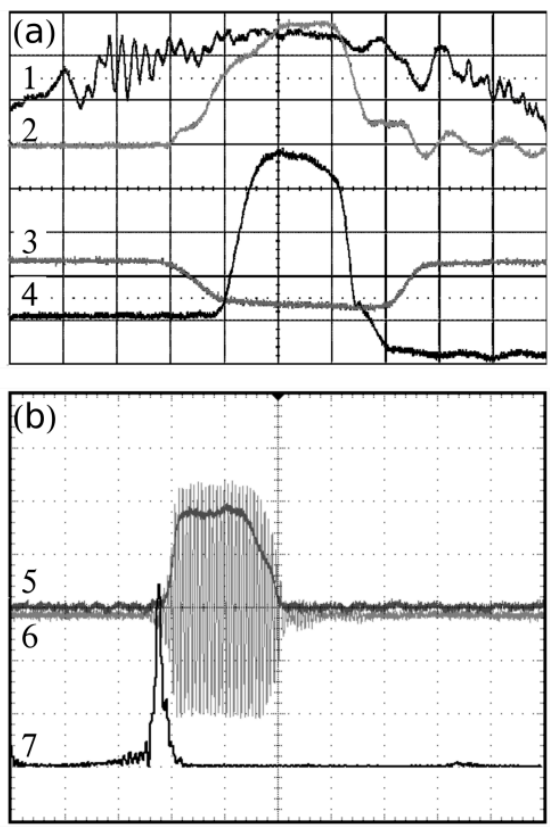

Fig. 3. Typical oscilloscope traces of (a) beam current at the accelerator output (curve 1) and the FEM-output (2), input driving signal from magnetron $(\mathrm{f} \approx 30 \mathrm{GHz})(3)$ and signal from the RF-power detector (4) as well as (b) output RF-pulse signal from the low-power detector (5), mixed heterodyne signal (6) and spectrum of the amplified signal (7): time scale $100 \mathrm{~ns} /$ div., frequency scale $50 \mathrm{MHz} /$ div

\section{FEM in a nonresonant trapping regime}

The second studied regime is the regime of the socalled non-resonant trapping [3, 4]. It utilizes a tapered wiggler (Fig. 4), in which the electrons are far from the resonance, $\omega>\left(2 \pi / d_{\mathrm{w} 0}+h\right) v_{\|}$, at the very beginning (line 1 in Fig. 4b). While the wiggler period $d_{\mathrm{w}}$ decreases along the interaction space (line 2 in Fig. 4b), the particles get trapped by the operating wave in a proximity of an inner resonance point and after that are efficiently decelerated. Though this regime requires twice as long interaction (compared to the regime of grazing), in simulations it provides a substantially higher amplification of up to $40 \mathrm{MW}$ and extremely wide amplification band up to $50 \%$, i.e. all over Ka-band.
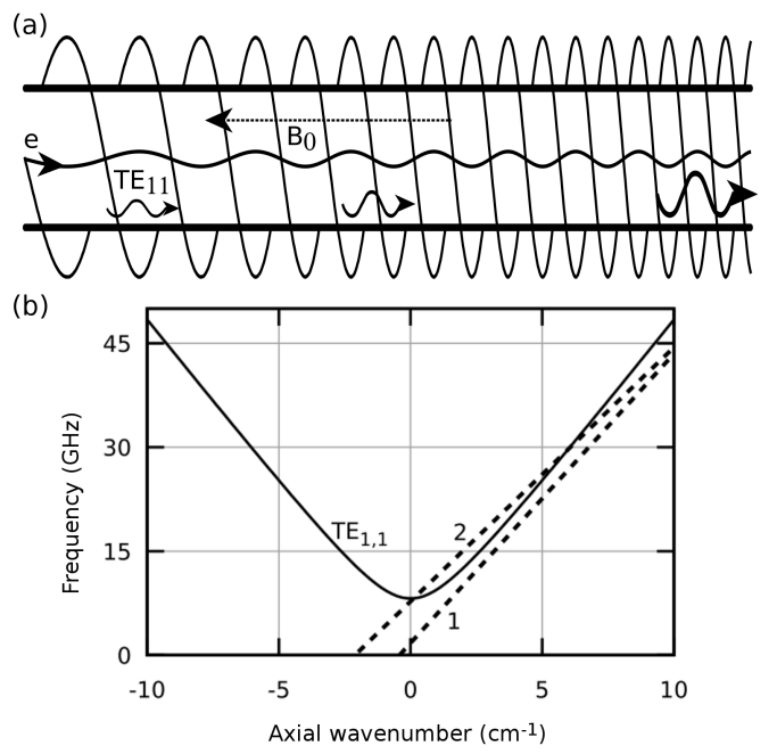

Fig. 4. (a) Schematic of the FEM amplifier with a profiled helical wiggler and (b) dispersion diagram for the $30-\mathrm{GHz}$ JINRIAP FEM-amplifier operated in the regime of nonresonant trapping. Solid and dashed lines show the dispersion of the $\mathrm{TE}_{1,1}$ wave and the electron beam, respectively

To provide the operation of the FEM-amplifier in the non-resonant trapping regime, a helical wiggler having the period profiled from $6.2 \mathrm{~cm}$ to $3.6 \mathrm{~cm}$ and the length of up to $2 \mathrm{~m}$ was designed. The undulator has been manufactured, and the magnetic field distribution measurements demonstrated a good coincidence with the results of electron-optical 3D simulations. Experimental studies of FEMamplifier of the novel type are currently in progress.

\section{Acknowledgments}

This work is partially supported by the Russian Government project \# 0035-2014-0012.

\section{References}

1. A.K. Kaminsky, E.A. Perelstein, S.N. Sedykh, e.a. Demonstration of powerful $30-\mathrm{GHz}$ FEM operation with resonant load // Tech. Phys. Lett. 2010. V. 36 No. 5 P. 37.

2. N.S. Ginzburg, I.I. Golubev, A.K. Kaminsky, e.a. Experiment on pulse heating and surface degradation of a copper cavity powered by powerful $30 \mathrm{GHz}$ free electron maser // Phys. Rev. Accel. Beams 2011. V. 14. No. 4. P. 041002.

3. A.V. Savilov, I.V. Bandurkin, N.Yu. Peskov. Regime of non-resonant trapping in an FEM-amplifier // Nucl. Instr. and Meth. in Phys. Res. A, 2003, 507, 158-161.

4. I.V. Bandurkin, A.K. Kaminsky, E.A. Perelstein, e.a. Modeling of a High-Power Wideband Free-Electron Maser Amplifier with an Operating Frequency of $30 \mathrm{GHz}$ to be Used in Particle Acceleration Experiments // Radiophys. and Quant. Electr. 2016. V. 58. No. 8. P. 607.

5. N.Yu. Peskov, I.V. Bandurkin, D.E. Donetc, e.a. Modeling of a High-Power Wideband Free-Electron Maser Amplifier with an Operating Frequency of $30 \mathrm{GHz}$ to be Used in Particle Acceleration Experiments // Appl. Phys. Lett. 2017. V. 110. P. 013501. 